\title{
Visualization of Optical Continuum Evolution Along a Nonlinear Waveguide
}

\author{
T. Chaipiboonwong ${ }^{1}$, J. D. Mills ${ }^{1}$, M. D. B Charlton ${ }^{2,3}$, M.E.Zoorob ${ }^{3}$, M.C.Netti, \\ J. J. Baumberg ${ }^{3,4}$, and W. S. Brocklesby ${ }^{1}$ \\ ${ }^{1}$ Optoelectronics Research Centre, University of Southampton, SO17 1BJ, UK, ${ }^{2}$ School of Electronics And Computer Science, University of \\ Southampton, SO17 1BJ, UK, ${ }^{3}$ Mesophotonics Ltd, Chilworth Science Park, Southampton, SO16 $7 N P$, UK, ${ }^{4}$ Dept of Physics and Astronomy, \\ University Of Southampton, SO17 IBJ, UK
}

\begin{abstract}
We describe the first non-destructive measurements of the evolution of the optical continuum along a non-linear waveguide. By utilizing near-field microscopy, the spectral variation can be imaged along and across the waveguide with sub-wavelength resolution.
\end{abstract}

OCIS codes: (180.5810) Scanning microscopy; (190.7110) Ultrafast nonlinear optics

Supercontinuum generation by ultrafast laser pulses using nonlinear effects in waveguides provide an important new light source for many areas of physics [1-3]. However, the understanding of the process of generating the supercontinuum is difficult because of the number of effects that contribute including self-phase modulation, soliton shedding, Raman scattering, and group velocity dispersion. To date, all study of these sources has been via their output only [4,5], but this information is necessarily limited. By using waveguides designed to allow access to the evanescent optical fields, and a Near-field Scanning Optical Microscope (NSOM), we can watch the generated continuum evolving along the length of the guide in a manner previously impossible. Here, we concentrate initially on the lower power regime - only a limited continuum bandwidth rather than the full supercontinuum.

The waveguide utilized in these experiments was chosen from a set of rib-waveguides on a Mesophotonics Ltd. supercontinuum generation chip. It consists of a $\mathrm{Ta}_{2} \mathrm{O}_{5}$ stripe of length $6 \mathrm{~mm}$, width $4 \mu \mathrm{m}$, and height $0.5 \mu \mathrm{m}$, on a layer of $\mathrm{SiO}_{2}$, grown on a silicon wafer. A schematic is shown in Figure 1(a). Laser pulses from a Coherent Mira oscillator of duration $86 \mathrm{fs}$, wavelength $800 \mathrm{~nm}$, energy per pulse $2.1 \mathrm{~nJ}$ at a repetition-rate of $76 \mathrm{MHz}$ were focused into the waveguide. The evanescent field of the optical mode which extended out into the air above the guide for

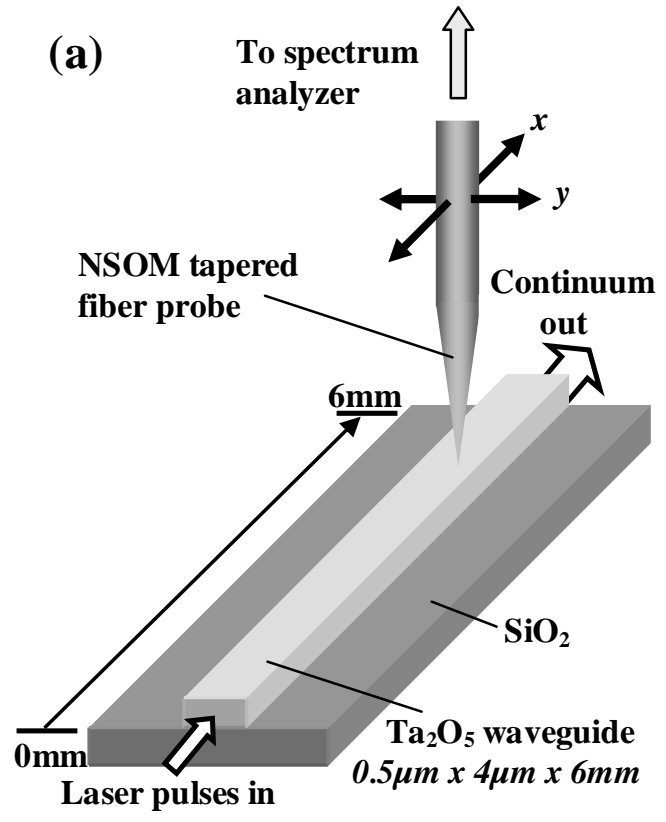

(b)

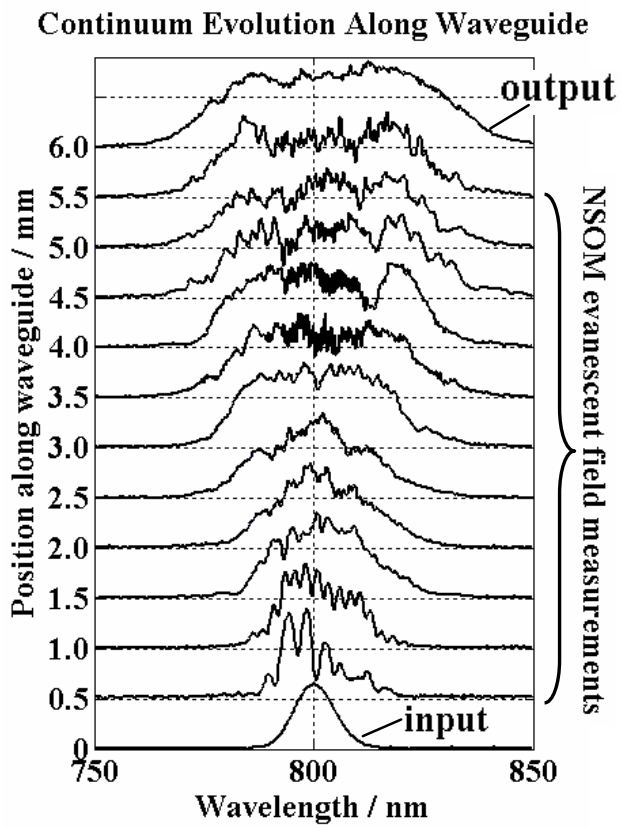

Fig. 1. (a) The NSOM probe is positioned at a height of $\sim 20 \mathrm{~nm}$ above the waveguide in order to sample the local spectrum via the evanescent field. (b) NSOM measured continuum growth as it evolves along the length of the waveguide (log scale). The spectra shown were obtained at $0.5 \mathrm{~mm}$ intervals along the central axis of the guide. Spectra shown at positions $0 \mathrm{~mm}$ and $6 \mathrm{~mm}$ show the pre-waveguide laser spectrum and postwaveguide output respectively. All other spectra shown from $0.5 \mathrm{~mm}$ to $5.5 \mathrm{~mm}$ represent NSOM evanescent-field measurements. 
$\sim 100 \mathrm{~nm}$ was sampled using an uncoated NSOM fiber probe of $\sim 80 \mathrm{~nm}$ tip diameter, held at a fixed distance of $20 \mathrm{~nm}$ from the guide surface by shear-force feedback [6]. The light collected by the tip was detected with a high-resolution CCD-based spectrometer. Figure 1(b) shows the spectrum of the continuum as it evolved along the length of the waveguide. The development of the continuum along the guide is clearly visible. The spectra shown were obtained along the central axis of the guide by sampling the local spectrum via the evanescent field at intervals of $0.5 \mathrm{~mm}$. The laser spectrum itself, having a FWHM of $11 \mathrm{~nm}$ is included in the figure at position 0mm for reference. Additionally, the waveguide output is shown at position $6 \mathrm{~mm}$. As can be seen, measurement at the guide output does not show the detailed spectral variation recorded by NSOM local sampling, which we have found to cycle over lengths of $\sim 5 \mu \mathrm{m}$ along the guide, caused by interference between modes. By repeating these measurements with different input powers we have studied how the continuum broadens under various conditions.

Figure 2 gives an example of how the spectrum of the generated light is observed to evolve on a much smaller length-scale. Here, measurements are shown in a direction along the guide. Spectral variation across the guide is also considerable as a consequence of modal beating. With the NSOM probe positioned at $3 \mathrm{~mm}$ along the guide's central axis, spectra were collected by stepping $100 \mathrm{~nm}$ intervals along an overall length of $2 \mu \mathrm{m}$. In this figure the spectrum is seen to broaden and narrow on a length scale of $\sim 400 \mathrm{~nm}$, which is approximately the wavelength of the light in the guide. We are currently extending these studies to include guides with greater confinement in order to simplify the modal structure and to seek understanding of the evolution of spectra in the regime of supercontinuum.

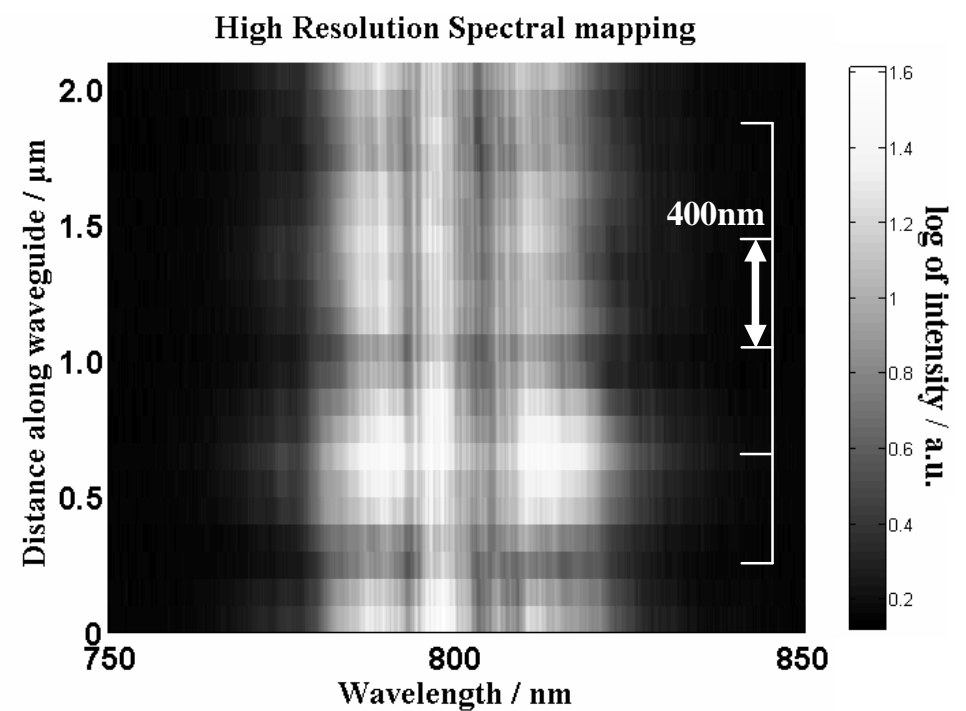

Fig. 2. Small-scale spatial variation of the spectrum measured at $100 \mathrm{~nm}$ intervals, at a distance $3 \mathrm{~mm}$ from the front end. The data was acquired by scanning along the central axis of the guide. The spectra are seen to broaden and narrow on a length scale of $\sim 400 \mathrm{~nm}$. The spatial variation was repeatable over periods of $\sim 1$ hour, demonstrating that the laser intensity and coupling into the guide was extremely stable, and did not contribute to the variations observed.

The capacity to visualize the development of nonlinear processes along waveguide devices with NSOM will not only enable a much better understanding of the important design properties of such devices, but also assist in the development of theory. This NSOM-based technique should also lend itself to more complex analysis of the nonlinear process, such as localized phase measurement or sub-wavelength-scale FROG analysis of the evolving pulse.

[1] R. Holzwarth, T. Udem, T.W. Hansch, J.C. Knight, W.J. Wadsworth, P.S.J. Russell, “Optical frequency synthesizer for precision spectroscopy", Phys. Rev. Lett. 85, 2264-2267, (2000)

[2] B.R. Washburn, S.A. Diddams, N.R. Newbury, J.W. Nicholson, M.F. Yan, C.G. Jorgensen, "Phase-locked, erbium-fiber-laser-based frequency comb in the near infrared", Opt. Lett. 29, 250-252 (2004)

[3] D.J. Jones, S.A. Diddams, J.K. Ranka, A. Stentz, R.S. Windeler, J.L. Hall, S.T. Cundiff, "Carrier-envelope phase control of femtosecond mode-locked lasers and direct optical frequency synthesis", Science 288, 635-639 (2000)

[4] Q. Cao, X. Gu, E. Zeek, M. Kimmel, R. Trebino, J. Dudley, R.S. Windeler, "Measurement of the intensity and phase of supercontinuum from an 8-mm-long microstructure fiber" Appl. Phys. B. 77, 239-244 (2003)

[5] T. Hori, N. Nishizawa, T. Goto, M. Yoshida, "Experimental and numerical analysis of widely broadened supercontinuum generation in highly nonlinear dispersion-shifted fiber with a femtosecond pulse", J. Opt. Soc. Am. B. 21, 1969-1980 (2004)

[6] Khaled Karrai, Robert D. Grober, "Piezoelectric tip-sample distance control for near field optical microscopes", Appl. Phys. Lett. 66, 18421844, (1995). 